\title{
Sobrecarga Emocional e Qualidade de Vida em Mães de Crianças com Erros Inatos do Metabolismo
}

\author{
Angela Cristina Pontes-Fernandes ${ }^{1}$ \\ Eucia Beatriz Lopes Petean \\ Universidade de São Paulo - Ribeirão Preto
}

\begin{abstract}
RESUMO - Este estudo objetivou investigar a sobrecarga emocional e a percepção da própria qualidade de vida em mães de crianças com Erros Inatos do Metabolismo (EIM). Participaram 21 mães de crianças com EIM, com comprometimento neurológico grave, de ambos os sexos, com idade até dez anos. A coleta de dados foi realizada por meio dos instrumentos WHOQOL-Bref e Burden Interview. Os dados foram analisados estatisticamente e comparados. Foi observado que os cuidados com a criança com EIM interferem negativamente na qualidade de vida das mães, sobretudo no domínio relações sociais, acarretando uma sobrecarga emocional, de tal forma que quanto maior a sobrecarga, mais baixa a percepção sobre a própria qualidade de vida.
\end{abstract}

Palavras-chave: doença crônica, sobrecarga emocional, qualidade de vida

\section{Emotional Burden and Quality of Life of Mothers with Children Having Inborn Errors of Metabolism}

\begin{abstract}
This study aims to investigate the perception of women having children with Inborn Errors of Metabolism (IEM) about the experienced emotional burden and their own quality of life. The participants were 21 mothers of children with IEM, with serious neurological injury. The children were of both sexes, up to ten years old. Data collection was accomplished through instruments WHOQOL-Bref and Burden Interview. The data were statistically analyzed and compared. The care of children with IEM interfere in a negative way in the quality of life of the mother, especially in the domain of their social relations, resulting in an emotional burden; the greater the burden, the lower the perceived quality of life.
\end{abstract}

Keywords: chronic disease, emotional burden, quality of life

Os Erros Inatos do Metabolismo (EIM) são doenças neurológicas crônicas, raras, determinadas geneticamente, que envolvem o metabolismo de substâncias químicas, decorrentes de alteração na informação contida no DNA recebido dos progenitores (Kok \& Diament, 2005). Sua manifestação pode-se dar através de um quadro de encefalopatia lenta e progressiva no recém-nascido, uma rápida deterioração clínica ao nascimento ou após um intervalo de boa saúde (Scriver, Beaudet, Sly \& Valle, 1995).

Apesar dos avanços no diagnóstico e tratamento dos EIM, para muitos casos o prognóstico se mostra pobre e o melhor que o médico pode oferecer às famílias é a possibilidade de prevenir o problema na ocorrência de uma futura gravidez (Levene, Chervenak \& Whittle, 2001).

A literatura é enfática ao afirmar o caráter disruptivo da doença crônica na dinâmica familiar (Castro \& Piccinini, 2002; Klaus, Kennel \& Klaus, 2000; Nereo, Fee \& Hinton, 2003; Pontes, Figueiredo, Gavioli \& Funayama, 2006). No caso do adoecimento de uma criança, estudos afirmam que, embora todos os membros da família sofram, é a figura materna que acaba por sofrer mais intensamente (Young, Dixon-Wood, Findlay \& Heney, 2002).

1 Endereço para correspondência: Rua Gonçalves de Magalhães, 490 Bairro Jardim Piratininga. Ribeirão Preto, SP. CEP 14030-570. Fone: (16) 3024-0987. Fax: (16) 3633-0866.E-mail: pontesfernandes.ac@ gmail.com
Não foi encontrada qualquer referência na literatura sobre estudos relacionando os EIM e suas repercussões emocionais no sujeito que adoece ou no sistema familiar. A grande maioria das pesquisas com estresse e sobrecarga de cuidadores se encontra na área geriátrica ou psiquiátrica (Sales, 2003). Em termos de qualidade de vida, os estudos abrangem diversas populações, no entanto, enfocam, em sua maioria, indivíduos portadores de alguma patologia (Dantas, Sawada \& Malerbo, 2003) e não seus cuidadores.

Definido pela Organização Mundial de Saúde (OMS), o conceito de qualidade de vida diz respeito à "percepção do indivíduo de sua posição na vida, no contexto da cultura e sistema de valores nos quais ele vive e em relação aos seus objetivos, expectativas, padrões e preocupações" (Fleck, et al, 2000, p. 179). Já, para a compreensão da noção de sobrecarga emocional (burden), destaca-se a definição de Cerqueira e Oliveira (2002), ou seja, problemas físicos, psicológicos ou emocionais, sociais e financeiros apresentados por cuidadores de pacientes dependentes.

A despeito da carência de estudos na área, pode-se entender que, tal como qualquer outra doença crônica, as doenças metabólicas afetam, não somente a criança acometida, mas também seus cuidadores. Neste sentido, o objetivo deste estudo foi investigar a sobrecarga emocional e a percepção da própria qualidade de vida em mães de crianças com EIM. 


\section{Método}

\section{Participantes}

Participaram deste estudo 21 mães de crianças com diagnóstico de EIM, com comprometimento neurológico grave, de ambos os sexos, com idade até dez anos, cuja manifestação da doença se deu até os três anos de idade da criança e há, pelo menos, dois anos.

Quanto à caracterização da amostra, as idades das mães participantes do estudo distribuíram-se na faixa compreendida entre 19 e 43 anos, sendo que $62 \%$ delas possuíam idade entre 30 e 39 anos. A grande maioria, 43\%, tinha nível de escolaridade que correspondia ao primeiro grau incompleto e apenas uma participante tinha formação superior. Noventa por cento das participantes viviam com companheiros (casadas ou em união estável) e 76\% não trabalhavam fora (“do lar"). A renda familiar média foi de 3,3 salários, sendo que $76 \%$ das participantes possuíam renda inferior a quatro salários. Vale ressaltar ainda que, das cinco mães que trabalhavam (24\%), a maioria o fazia por meio período ou sem horário pré-determinado, de modo a conciliar a ocupação com os cuidados dispensados ao filho com EIM.

Em relação às crianças selecionadas para o estudo, 71\% eram do sexo masculino, sendo que a grande maioria, $67 \%$, ocupava a posição de caçula na família e $28 \%$ eram filhos únicos. Quanto ao início de aparecimento dos sintomas, em $48 \%$ dos casos a sintomatologia surgiu nos primeiros meses de vida da criança.

Como procedimento para seleção das participantes foi utilizada a entrevista clínica estruturada para DSM III-R não paciente (SCID-NP), descrita originalmente por Spitzer, Willians \& First (1989) e adaptada para o português por Del Bem (1995), de modo a excluir da amostra participantes com indícios de sintomas psicóticos, tais como: perda do vínculo com a realidade, alucinação, delírio.

A seleção das participantes foi feita no Ambulatório de Erros Inatos do Metabolismo (AEIM), do Hospital das Clínicas da Faculdade de Medicina de Ribeirão Preto - Universidade de São Paulo (HCFMRP-USP), a partir do levantamento dos casos atendidos entre os anos de 2000 a 2006 e o auxílio da médica responsável pelo ambulatório.

Os EIM destacados no presente trabalho podem ser classificados em cinco grandes grupos: as acidemias orgânicas, as desordens do ciclo da ureia, as doenças lisossomais, as leucodistrofias e outras desordens. A despeito das particularidades de cada diagnóstico, em todos os casos o comprometimento neurológico era grave, levando a um significativo nível de dependência por parte das crianças. De maneira geral, os sintomas compartilhados eram: involução das aquisições, atraso no desenvolvimento neuropsicomotor, hipotonia e convulsões. Os tratamentos concentram-se no alívio dos sintomas por meio de dietas ou medicamentos (Kok \& Diament, 2005; Levene, Chervenak \& Whittle, 2001).

\section{Instrumentos}

Foram utilizados os seguintes instrumentos: (a) Questionário "World Health Organization Quality of Life”, em sua versão abreviada, WHOQOL-Bref, (Fleck et al., 2000). Trata-se de um instrumento de avaliação da qualidade de vida, que conta com 26 questões e é subdividido em quatro domínios: físico, psicológico, relações sociais e meio ambiente; e (b) Escala Burden Interview (BI). É um instrumento para a avaliação da sobrecarga, adaptado para a população brasileira por Scazufca (2002), que conta com 22 itens no total, dos quais, 21 devem ser respondidos através de uma escala do tipo Likert, de cinco itens dispostos em progressão positiva, e um último item geral, no qual o respondente é questionado sobre o quanto ele se sente sobrecarregado com a atribuição de cuidador.

\section{Procedimento}

Esta pesquisa foi aprovada pelo Comitê de Ética em Pesquisa Envolvendo Seres Humanos do Hospital das Clínicas da Faculdade de Medicina de Ribeirão Preto, da Universidade de São Paulo (HCFMRP-USP).

A coleta de dados foi realizada em um encontro com cada participante, em sua maioria no próprio hospital (uma única mãe foi visitada em sua casa). Neste encontro, os instrumentos foram aplicados pela pesquisadora, que os lia integralmente para as participantes e anotava suas respostas no próprio protocolo. O contato inicial se deu por telefone e/ ou pessoalmente. Todas as participantes assinaram o Termo de Consentimento Livre e Esclarecido.

Visando a garantia do anonimato das mães e seus filhos, as duplas foram numeradas e identificadas com as letras $\mathrm{M}$ (mãe) e C (criança).

\section{Análise dos dados}

A análise dos dados obtidos pelo WHOQOL-Bref se fez por meio do programa estatístico SPSS for Windows versão 6.0, de acordo com as recomendações específicas da literatura especializada (Fleck et al., 2000). Com o objetivo de estabelecer parâmetros para a localização dos sujeitos no conjunto dos resultados da população estudada, obtidos nos quatro domínios do instrumento, foram realizados estudos descritivos, através da verificação da mediana, valores máximos e mínimos.

Os dados da BI foram pontuados de acordo com as normas da adaptação para a população brasileira descritas por Scazufca (2002) e, tal como no caso do WHOQOL-Bref, foram analisados em termos de parâmetros descritivos.

A conjugação dos dados dos instrumentos entre si foi feita por meio da Técnica de Quadrantes descrita por Figueiredo (1994) e utilizada por Souza (2001) e Saviani-Zeoti \& Petean (2008). Os quadrantes são definidos nos diagramas de dispersão, a partir das medianas dos eixos X e Y. Deste modo, para o cruzamento do WHOQOL-Bref com a BI, os quadrantes foram definidos a partir da mediana desta última, disposta nas abscissas, e das medianas da distribuição dos índices de satisfação frente aos domínios do WHOQOL-Bref, nas ordenadas. Os pontos de corte (medianas) subdividem os diagramas de dispersão em quatro campos. 
A Figura 1 exemplifica a forma de análise e interpretação dos resultados, por meio da conjugação entre os dados sobre a qualidade de vida (QV) e a sobrecarga.

Com base neste diagrama, cada participante pode ser localizada em um dos quatro quadrantes, a partir da conjuga-

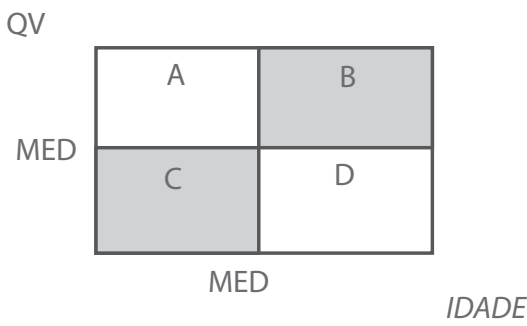

Figura 1. Esquema de Quadrantes.

ção das medianas da Sobrecarga e do índice de Qualidade de Vida. A interpretação da Análise de Quadrantes, para o estudo das conjugações entre as medidas estabelecidas, é dada da seguinte maneira: (a) Quadrante A - QV acima da mediana associada à Sobrecarga abaixo da mediana; (b) Quadrante B - QV acima da mediana associada à Sobrecarga acima da mediana; (c) Quadrante C - QV abaixo da mediana associada à Sobrecarga abaixo da mediana; (d) Quadrante D - QV abaixo da mediana associada à Sobrecarga acima da mediana.

A localização de cada participante em cada um dos diagramas possibilitou a discriminação dos quadrantes prevalentes com base em estudos de diferenças de proporções tendo-se como critério $\mathrm{p} \leq 0,005(\mathrm{Pz}>1,96)($ Souza, 2001).

\section{Resultados}

O tratamento dos dados obtidos pelo WHOQOL-Bref resultou nas medianas dos índices de satisfação das participantes, para cada um dos quatro domínios avaliados (Figura 2).

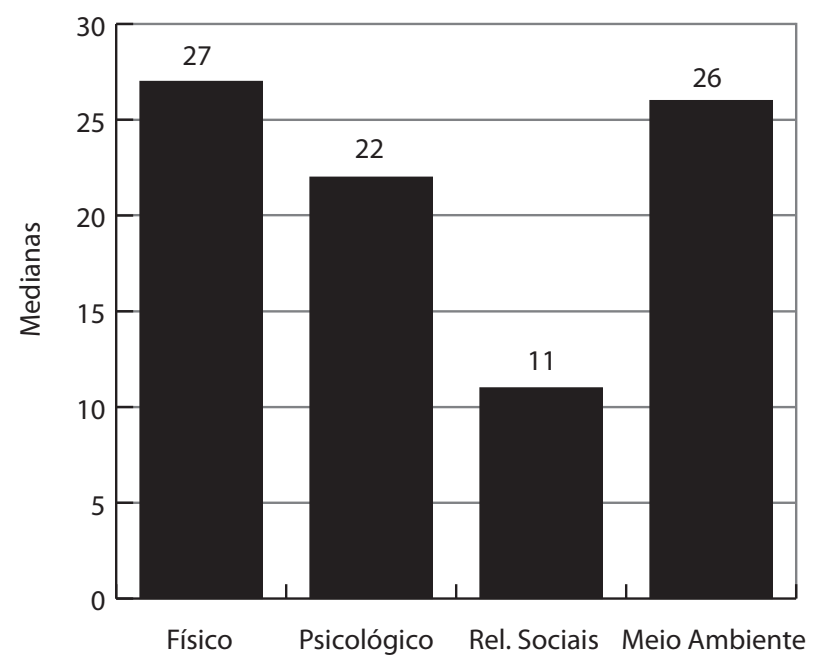

Figura 2. Mediana da distribuição dos índices de satisfação das participantes, frente aos domínios do WHOQOL-Bref, em relação ao conjunto das participantes
De acordo com a Figura 2, em termos de qualidade de vida, houve uma maior satisfação com os aspectos que integram os domínios físico e meio ambiente, bem como um menor índice de satisfação em relações sociais.

$\mathrm{O}$ tratamento dos dados obtidos pela Burden Interview seguiu as normas da escala, através do somatório das avaliações frente a cada uma das participantes (Figura 3), obtendo-se, assim, a distribuição dos valores totais em relação a este instrumento.

Na Figura 3, observa-se que na população estudada há mães que se destacam por suas pontuações elevadas (M2, M11 e M15), no instrumento que avalia o grau de sobrecarga vivenciado em virtude dos cuidados oferecidos à criança, $\mathrm{e}$ outras três participantes que se destacam por suas pontuações rebaixadas (M7, M8 e M14). A mediana da sobrecarga apresentada pelas participantes foi de 25 , distribuída entre uma pontuação máxima de 40 e uma mínima de 14 .

Considerando que a BI apresenta um escore máximo de 88 pontos, observa-se que a pontuação máxima (40) apresentada pelas participantes encontrava-se abaixo da média prevista pelo instrumento (44). Apesar disto, uma análise qualitativa das respostas dadas ao instrumento mostra que, com relação às questões referentes à dependência da criança e dedicação exclusiva ao filho com EIM, as mães demonstraram sentir o peso da sobrecarga decorrente da condição de cuidadoras: "sempre" $(85,7 \%)$ sentem que a criança depende delas; "algumas vezes" $(52,4 \%)$ sentem que não têm tempo para si ou se sentem estressadas ao somarem a função de cuidadora com suas outras responsabilidades; e ainda, "algumas vezes" $(52,9 \%)$ têm dúvidas sobre o que fazer pela criança.

Os dados referentes ao índice de sobrecarga vivenciada pelas mães foram conjugados com a variável qualidade de vida, por meio da Técnica de Quadrantes (Figueiredo, 1994).

Seguindo o método utilizado por Souza (2001), para a conjugação dos dados do WHOQOL-Bref com os resultados da Burden Interview, os quadrantes foram definidos pelo posicionamento das medianas das distribuições dos índices de satisfação das participantes, frente aos domínios que constituem o instrumento de qualidade de vida, nas ordenadas, e da distribuição do índice de sobrecarga das mesmas participantes, nas abscissas. Através deste método de análise, foi possível identificar as relações entre a qualidade de vida das mães e a sobrecarga sentida por elas nas atividades de cuidado com a criança (Figura 4):

Através destes resultados, pode-se observar que a sobrecarga sentida pelas mães nos cuidados com as crianças apresenta uma relação inversamente significativa com todos os domínios do WHOQOL-Bref. As mães que se sentem mais sobrecarregadas nos cuidados com seu filho apresentam-se menos satisfeitas com os fatores de bem-estar físico e psicológico, relacionamentos sociais e meio ambiente, relacionados com a sua qualidade de vida.

\section{Discussão}

Os resultados deste estudo possibilitaram conhecer alguns aspectos da sobrecarga emocional em mães cuidadoras de filhos com EIM e a percepção das mesmas sobre a interferência desta situação em sua qualidade de vida. 


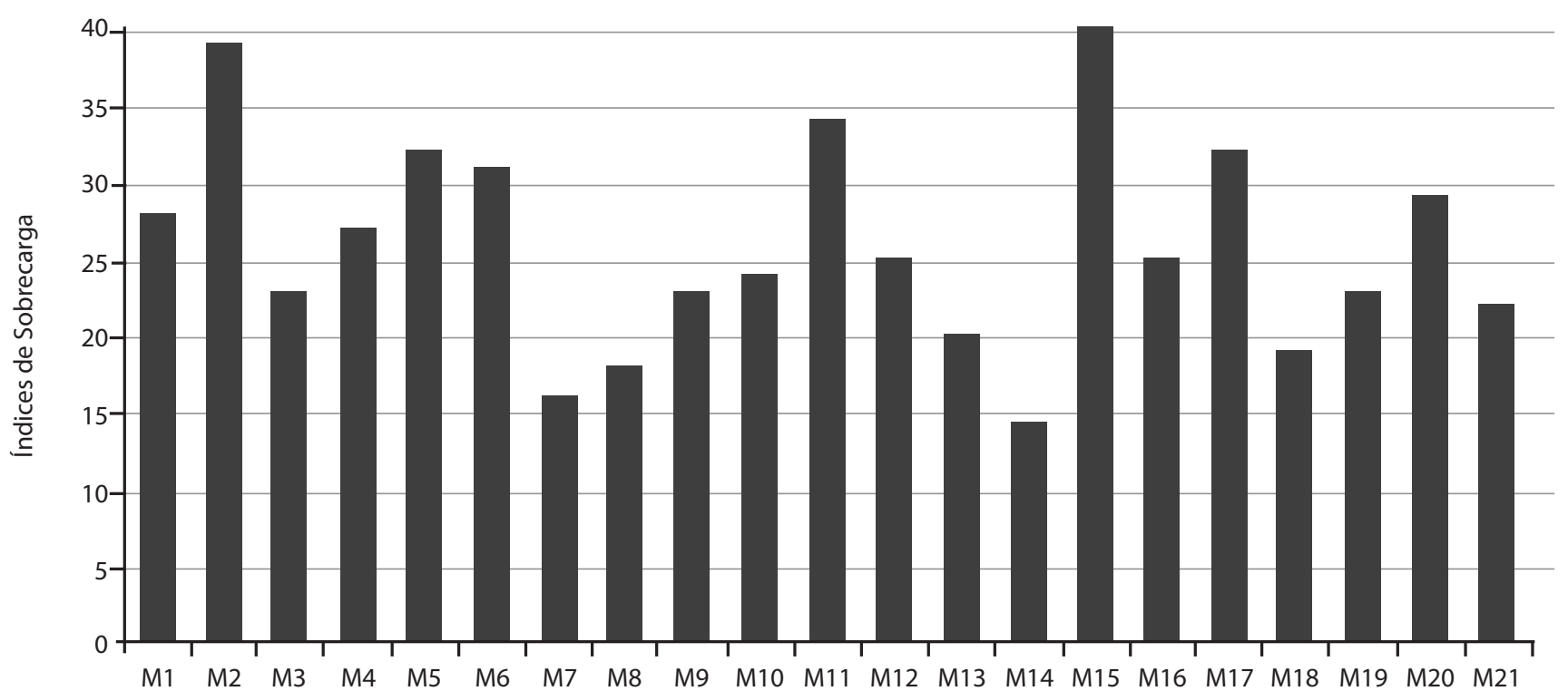

Figura 3. Distribuição da sobrecarga total para cada uma das participantes, obtida por meio do instrumento BI

WHOQOL - Bref (Físico)
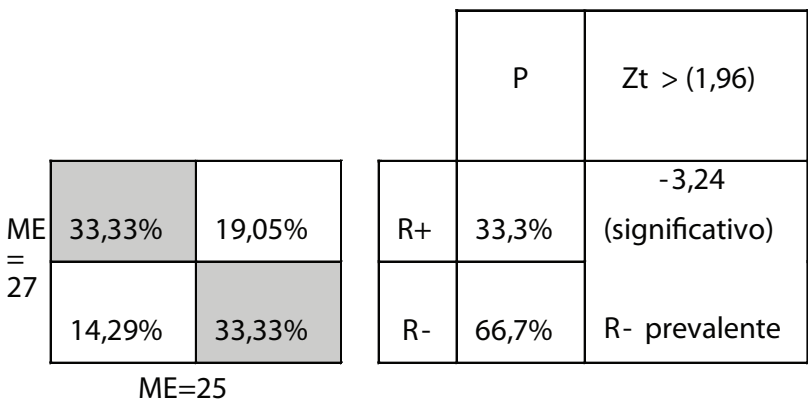

Sobrecarga
WHOQOL - Bref (Psicológico)
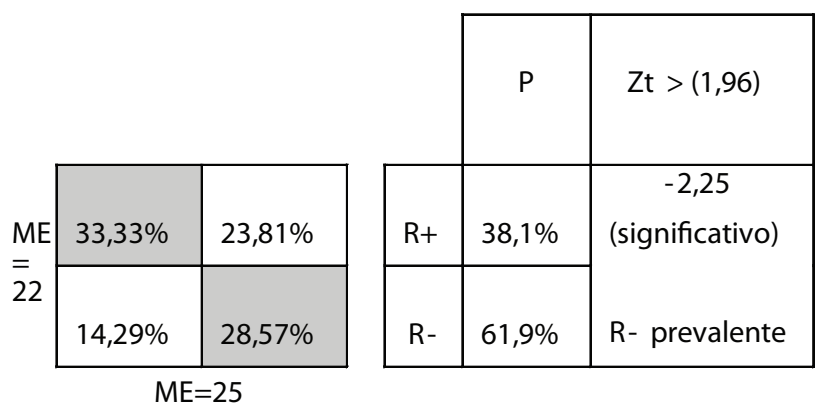

Sobrecarga

WHOQOL - Bref (Relações Sociais)

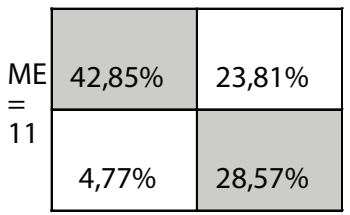

$\mathrm{ME}=25$

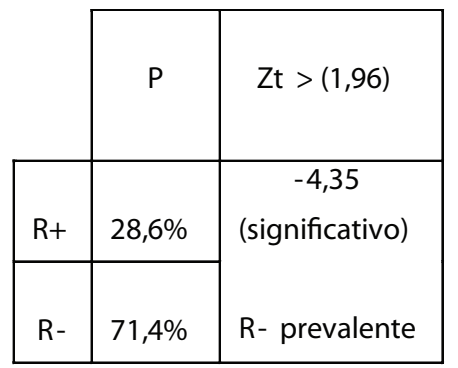

Sobrecarga
$\mathrm{ME}=25$

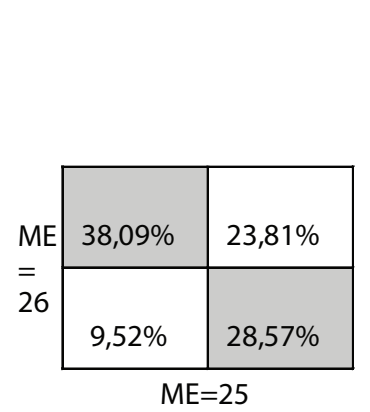

WHOQOL - Bref (Meio Ambiente)

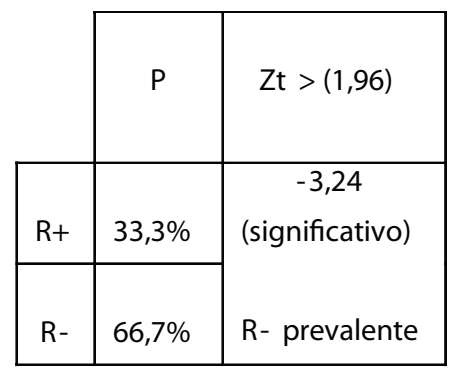

Sobrecarga

Figura 4. Conjugação dos índices do WHOQOL-Bref, para os quatro domínios, com os resultados da BI.

A raridade dos quadros de EIM associada aos critérios de inclusão estabelecidos nesta pesquisa propiciou o acesso a toda população de mães, cujas crianças foram acompanhadas pela instituição de saúde escolhida, durante um período de seis anos. Deste modo, a amostra se constituiu de toda a população disponível e que aceitou fazer parte do estudo.

Considerando os instrumentos utilizados nesta pesqui$\mathrm{sa}$, os dados de literatura indicam que muito pouco tem sido estudado sobre a sobrecarga e qualidade de vida em cuidadores de crianças com problemas neurológicos. No Brasil, o WHOQOL-Bref tem sido utilizado para avaliar a qualidade de vida, sobretudo, em pacientes (Berlim \& Fleck, 2003; Fleck, Chachamovich \& Trentini, 2003; Michelone \& Santos, 2004; Morales, 2005; Zalihic, Markotic, Zalihic \& Mabic, 2010), sendo que, até o presente momento, pouco tem sido estudado a respeito da qualidade de vida de cuidadores. 
Alguns estudos utilizando o WHOQOL-Bref em cuidadores são: Vikas, Avasthi e Sharan (2011), que avaliou cuidadores de pacientes com desordem obsessivo-compulsiva; Romeo et al. (2010), que estudou a qualidade de vida em pais de crianças com paralisia cerebral; Lin et al. (2009), que avaliou cuidadores de crianças e adolescentes com inabilidades intelectuais; Schestatsky et al. (2006), que avaliou cuidadores de pacientes com doença de Parkinson, e Westphal et al. (2005), com cuidadores de pessoas com epilepsia.

Dantas, Sawada e Malerbo (2003) confirmam esta escassez de estudos brasileiros com a população de cuidadores, uma vez que a maioria dos estudos prioriza a avaliação do paciente apenas.

No que diz respeito à $\mathrm{BI}$, o levantamento bibliográfico realizado, apontou dois estudos brasileiros utilizando este instrumento: Westphal et al. (2005), com cuidadores de pacientes epilépticos, e Garrido e Menezes (2004), que avaliaram o impacto em cuidadores de pacientes demenciados.

No caso da avaliação da qualidade de vida, embora não haja normas brasileiras para a comparação, os dados do WHOQOL-Bref sugeriram que a satisfação com relação à própria qualidade de vida, nas mães de crianças com EIM que participaram deste estudo, está baixa para os quatro domínios avaliados. Neste sentido, Young et al. (2002) afirmam que mães que assumem o papel de cuidar necessitam lidar com diversas e complexas obrigações para com a criança doente e os resultados de seu estudo sugerem que " $[. .$.$] administrar$ essas obrigações causou muitos efeitos adversos na qualidade de vida das mães" (Young et al. , 2002, p. 1844).

Neste estudo, o domínio que obteve o mais baixo índice foi relações sociais, indicando uma menor satisfação das participantes com suas relações pessoais, suporte/apoio social e atividade sexual. Possivelmente, isto está relacionado com a dedicação quase que exclusiva, por parte das mães, para com seus filhos, privando-as de cultivar e usufruir de uma vida social com amigos e do próprio relacionamento conjugal. Sobre isto, Irvin, Klaus e Kennel (1993) afirmam, em seu trabalho sobre pais de bebês com malformação congênita, que, especialmente as mães, sentem a necessidade de proteger o filho, isolando-os, muitas vezes, do convívio social.

Os domínios físico e meio ambiente do WHOQOL-Bref, apesar de apresentarem baixos índices, foram aqueles em que as participantes se mostraram mais satisfeitas. Isto sugere que os prejuízos psicológicos (emocionais) e sociais envolvidos nos cuidados do filho tomam tamanha proporção em suas vidas que podem suplantar as dificuldades envolvidas nos outros aspectos da vida, como o bem-estar físico e meio ambiente.

Pode-se supor que uma maior satisfação no domínio físico se deva tanto à baixa média de idade entre as participantes, 32 anos, que, por isso, ainda podem contar com o vigor da juventude, quanto à negligência, geralmente demonstrada pelas mães, no cuidado de si mesmas, em função da rotina de cuidados com o filho doente, prejudicando, ou até mesmo suprimindo, a percepção de seu próprio bem-estar físico (Young et al., 2002).

Ao se avaliar a sobrecarga das mães participantes do estudo, através da Burden Interview, pôde-se constatar que os índices encontraram-se na faixa entre leve e moderada, quando comparados com outros estudos que utilizaram o mesmo instrumento. Garrido e Menezes (2004) encontraram uma média moderada de sobrecarga entre cuidadores de idosos com demência e Westphal et al. (2005) consideraram de leve a moderada a sobrecarga encontrada em cuidadores de pacientes com epilepsia. Os índices de ambos os estudos, todavia, mostraram-se superiores aos desta pesquisa. Além disto, no item geral, no qual o respondente é questionado sobre o quanto ele se sente sobrecarregado com a atribuição de cuidador, mais da metade das participantes respondeu que "um pouco" ou "nem um pouco".

Diante dos diversos estudos aqui mencionados sobre o impacto inerente à função de cuidar de alguém com doença crônica, sobretudo, no caso de uma mãe com seu filho, chama a atenção o baixo índice de sobrecarga encontrado nesta pesquisa.

Uma hipótese explicativa para os baixos índices de sobrecarga encontrados neste estudo, defendida por alguns autores (Green, 2006; Pickett, Cook, Cohler \& Solomon, 1997; Westphal et al., 2005), seria a percepção, não somente de uma sobrecarga, mas, principalmente, de uma gratificação no cuidar, suplantando os prejuízos advindos desta função.

Ao responderem a um instrumento estruturado, cujas questões abordam explicitamente o tema da sobrecarga, as mães relataram nunca sentir que a criança afeta negativamente sua privacidade, saúde física, emocional, relacionamentos ou vida social, e ainda, em sua maioria, descartaram a possibilidade, mesmo que no plano do desejo, de um dia deixar de cuidar da criança por algum motivo.

Neste sentido, acredita-se que o caráter estruturado do instrumento, em sua abordagem direta do tema da sobrecarga, pode ter contribuído para inibir o julgamento das participantes sobre as próprias dificuldades na relação com o filho e sua doença, bem como os sentimentos evocados por esta situação, de modo que a possibilidade do desvelamento destes aspectos ficou prejudicada. Levanta-se também a hipótese de que esta inibição propiciada pelo instrumento psicométrico seja, na verdade, a externalização de um "esforço" das participantes, consciente ou mesmo inconsciente, de não entrar em contato com tais sentimentos conflitantes.

Considera-se, ainda, que o baixo índice de sobrecarga encontrado neste estudo possa ter decorrido do fato de que, embora o impacto exista, ele não pode ser reconhecido e, muito menos, verbalizado, uma vez que vivemos em uma cultura em que é difícil para uma mãe admitir que um filho seja um peso, uma sobrecarga em sua vida.

Embora Garrido e Menezes (2004, p. 839) tenham encontrado que "[...] indivíduos da família nuclear apresentaram maiores níveis de impacto que outros" no cuidado de pessoas com doenças crônicas, alguns trabalhos enfatizam que, no caso de crianças, o peso desta função acaba recaindo sobre a mãe. Trata-se da questão do gênero nas relações de cuidado como um padrão cultural, que atribui à mãe o papel de incondicionalidade e, geralmente, exclusividade nos cuidados para com o filho doente (Badinter, 1985; Dellve, Samuelsson, Tallborn, Fasth \& Hallberg, 2006; Castro \& Piccinini, 2002; Silva \& Correa, 2006).

Neste sentido, considera-se que os baixos índices quantitativos de sobrecarga aqui encontrados corroboram os dados dos estudos acima citados, na medida em que às mães não seria "culturalmente permitido" assumir, conscientemente, a 
sobrecarga vivenciada nos cuidados com um filho acometido por uma doença incurável.

Vale ressaltar que, apesar do baixo índice geral de sobrecarga encontrado, algumas questões do instrumento psicométrico exibiram escores acima do ponto médio (acima de 44 pontos), sinalizando, de alguma maneira, o impacto do adoecimento da criança na vida destas mães. As questões do instrumento que refletiram vivências de sobrecarga dizem respeito à constatação da dependência do filho para com seus cuidados, ao medo pelo futuro da criança, à necessidade de cuidar mais e melhor do filho, à falta de tempo para si mesma e ao estresse diante da difícil conciliação entre a função de cuidadora e suas outras responsabilidades com a família e demais atribuições do lar ou trabalho. Preocupações estas, esperadas no caso de mães de crianças com doenças crônicas.

Sobre isto, Bandeira e Barroso (2005) concluíram por uma relação entre a sobrecarga do cuidador e a sintomatologia da doença do paciente, o excesso de tarefas nos cuidados e suas outras responsabilidades, bem como a preocupação com o bem-estar presente e futuro do paciente.

Alguns dados da caracterização da amostra contribuem para confirmar a existência desta sobrecarga não declarada. Um deles é o reduzido número de mães que relataram trabalhar (apenas cinco) e o fato de todas elas exercerem atividades que permitiam um horário flexível ou apenas por meio período do dia, de modo a conciliar a ocupação com os cuidados dispensados ao filho. Outro aspecto importante a ser destacado neste caso é o dado de que $95 \%$ das crianças ou eram filhos únicos ou caçulas, o que pode sugerir a desistência de novos filhos após o nascimento da criança com EIM.

Diante do exposto, considera-se que, embora exista uma sobrecarga que recai sobre a mãe cuidadora de um filho com EIM, ela permanece velada, escondida aos olhos menos atentos, sob pena de que sua manifestação traga consigo emoções e sentimentos ambivalentes, difíceis de serem admitidos e elaborados.

\section{Considerações Finais}

A avaliação da qualidade de vida representou importante fonte de informação no que se refere à constatação de uma menor satisfação das mães, sobretudo, com os fatores de relacionamento social (relações pessoais, suporte/apoio social e atividade sexual). Os aspectos da sobrecarga percebida após o adoecimento da criança também revelaram uma relação inversamente proporcional com sua qualidade de vida, em todos os domínios avaliados.

Conclui-se que o impacto do adoecimento da criança e a administração de todas as obrigações inerentes a esta situação causam efeitos adversos na qualidade de vida das mães, bem como sobrecarga emocional. Contudo, muitas vezes, a sobrecarga vivenciada não pode ser expressa e reconhecida de maneira consciente, o que alimenta a falsa ideia de que não é penoso ser mãe e cuidar de uma criança com os sintomas de um dos mais raros e graves grupos de doenças neurológicas que atingem a infância.
Vale ressaltar que, embora este estudo tenha incluído toda a população de mães, cujos filhos foram acompanhados na instituição de saúde escolhida, durante seis anos, o reduzido número da amostra $(n=21)$ aponta para a necessidade de cautela na generalização dos resultados e conclusões aqui levantados.

Sobre o instrumento de avaliação da sobrecarga, faz-se importante destacar o fato de que, a despeito de sua validade e inegável contribuição para o presente estudo, seus resultados, na situação específica de avaliação da sobrecarga de mães de crianças com EIM, devem ser analisados com crítica e cautela.

Considera-se que as informações obtidas nesta investigação podem contribuir para uma maior compreensão da sobrecarga emocional e qualidade de vida em mães cuidadoras de crianças com doenças crônicas e degenerativas, com vistas à promoção de intervenções terapêuticas sensíveis às características e necessidades específicas desta população, bem como subsidiar o desenvolvimento de serviços de saúde mais preparados para lidar, não somente com a criança com EIM, mas também com sua família e, mais especificamente, com suas mães.

\section{Referências}

Badinter, E. (1985). Um amor conquistado: o mito do amor materno. Rio de Janeiro: Nova Fronteira.

Bandeira, M., \& Barroso, S. M. (2005). Sobrecarga das famílias de pacientes psiquiátricos. Jornal Brasileiro de Psiquiatria, $54,34-46$.

Berlim, M. T., \& Fleck, M. P. A. (2003). Quality of life: a brand new concept for research and practice in psychiatry. Revista Brasileira de Psiquiatria, 25, 249-252.

Castro, E. K., \& Piccinini, C. A. (2002). Implicações da doença orgânica na infância para as relações familiares: algumas questões teóricas. Psicologia: Reflexão e Crítica, 15, 625-635.

Cerqueira, A. T. A. R., \& Oliveira, N. I. L. (2002). Programa de apoio a cuidadores: uma ação terapêutica e preventiva na atenção à saúde dos idosos. Psicologia USP, 13, 1-12.

Dantas, R. A. S., Sawada, N. O., \& Malerbo, M. B. (2003). Pesquisas sobre qualidade de vida: revisão da produção científica das universidades públicas do Estado de São Paulo. Revista Latinoamericana de Enfermagem, 11, 532-538.

Del Bem, C. M. (1995) Estudo da confiabilidade do diagnóstico psiquiátrico obtido através da Entrevista Clínica Estruturada para o DSM-III-R (SCID) em serviço ambulatorial de um hospital escola. Dissertação de Mestrado, Universidade de São Paulo, Ribeirão Preto.

Dellve, L., Samuelsson, L., Tallborn, A., Fasth, A.,, \& Hallberg, L. R. (2006). Stress and well-being among parents of children with rare diseases: a prospective intervention study. Journal of Advanced Nursing, 53, 392-402.

Figueiredo, M. A. C. (1994) Profissionais de saúde e atitudes frente a sindrome da imunodeficiência adquirida: um estudo diferencial com base no modelo afetivo-cognitivo de Fishbein e Ajzen (1975). Tese de Livre-docência, Universidade de São Paulo, Ribeirão Preto. 
Fleck, M. P., Lealz, O. F., Louzada, S.; Xavier, M., Chachamovich, E., Vieira, G., Santos, L., \& Pinzon, V. (2000). Aplicação da versão em português do instrumento abreviado de avaliação da qualidade de vida "WHOQOL-bref". Revista de Saúde Pública, 34, 178-183.

Fleck, M. P. A., Chachamovich, E., \& Trentini, C. M. (2003). Projeto WHOQOL-OLD: método e resultados de grupos focais no Brasil. Revista de Saúde Pública, 37, 793-799.

Garrido, R., \& Menezes, P. R. (2004). Impacto em cuidadores de idosos com demência atendidos em um serviço psicogeriátrico. Revista de Saúde Pública, 38, 835-841.

Green, S. E. (2006). "What do you mean 'what's wrong with her?": stigma and the lives of families of children with disabilities. Social Science \& Medicine, 57, 1361-1374.

Irvin, N. A., Klaus, M. H., \& Kennel, J. H. (1993) Atendimento aos pais de bebês com malformação congênita. In M. H., Klaus \& J. H. Kennell (Eds.), Pais bebês a formação do apego. Porto Alegre, RS: Artes Médicas.

Klaus, M. H., Kennel, J. H., \& Klaus, P. H. (2000). Vinculo: construindo as bases para um apego seguro e para a independência. Porto Alegre: Artmed.

Kok, F., \& Diament, (2005) A.. Erros inatos do metabolismo: generalidades e mecanismos. In A. Diament \& S. Cypel (Eds.), Neurologia infantil. São Paulo, SP: Atheneu.

Levene, M. I., Chervenak, F. H., \& Whittle, M. (2001). Fetal and neonatal neurology and neurosurgery. London: Churchill Livingstone.

Lin, J. D., Hu, J., Yen, C. F., Hsu, S. W., Lin, L. P., Loh, C. H., Chen, M. H., Wu, S. R., Chu, C. M., \& Wu, J. L. (2009). Quality of life in caregivers of children and adolescents with intellectual disabilities: use of WHOQOL-BREF survey. Research in Developmental Disabiitiesl, 30, 1448-1458.

Michelone, A. P. C., \& Santos, V. L. C. G. (2004). Qualidade de vida em adultos com câncer colorretal com e sem ostomia. Revista Latino-Americana de Enfermagem, 12, 875-883.

Morales, N. M. O. (2005). Avaliação transversal da qualidade de vida em crianças e adolescentes com paralisia cerebral por meio de um instrumento genérico (CHQ-PF50). Dissertação de Mestrado, Universidade Federal de Uberlândia, Uberlândia.

Nereo, N. E., Fee, R. J., \& Hinton, V. J. (2003). Parental stress in mothers of boys with Duchenne Muscular Dystrophy. Journal of Pediatric Psychology, 28, 473-484.

Pickett, S. A., Cook, J. A., Cohler, B. J., \& Solomon, M. L. (1997). Positive parent/adult child relationships: impact of severe mental illness and caregiving burden. American Journal of Orthopsychiatry, 67, 67-72.

Pontes, A. C., Figueiredo, L. U. R., Gavioli, N. B., \& Funayama, C. R. A. (2006). Multidisciplinary approach to hospitalized epileptic children and their families. Einstein, 4, 89-94.

Romeo, D. M., Cioni, M., Distefano, A., Battaglia, L. R., Costanzo, L., Ricci, D., De Sanctis, R., Romeo, M. G., Mazzone, D., \& Mercuri, E. (2010). Qualty of life in parents of children with cerebral palsy: is it influence by the child's behaviour? Neuropediatrics, 41, 121-126.
Sales, E. (2003). Family burden and quality of life. Quality of Life Research, 12, 33-41.

Saviani-Zeoti, F. \& Petean, E. B. L. (2008). A qualidade de vida de adultos com deficiência mental leve, na percepção destas pessoas e na de seus cuidadores. Psicologia: Teoria e Pesquisa, 24, 305-311.

Scazufca, M. (2002). Brazilian version of the Burden Interview scale for the assessment of burden of care in careers of people with mental illnesses. Revista Brasileira de Psiquiatria, 24, 12-17.

Scriver, C. R., Beaudet, A. L., Sly, W. S., \& Valle, D. (1995). The metabolic and molecular bases of inherited disease (Vol. 1, 7th ed.). New York: McGraw-Hill.

Schestatsky, P., Zanatto, V. C., Margis, R., Chachamovich, E., Reche, M., Batista, R. G., Fricke, D., \& Rieder, C. R. M. (2006). Quality of life in a Brazilian sample of patients with Parkinson's disease and their caregivers. Revista Brasileira de Psiquiatria, 28, 209-211.

Silva, F. M., \& Correa, I. (2006). Doença crônica na infância: vivência do familiar na hospitalização da criança. Reme: Revista Mineira de Enfermagem, 10, 18-23.

Souza, L. B. (2001). Organização do trabalho, qualidade de vida e subjetividade: um estudo sobre representações e crenças de profissionais do campus da USP de Ribeirão Preto. Tese de Doutorado, Universidade de São Paulo, Ribeirão Preto.

Spitzer, R. L., Williams, J. B., \& First, M. B. (1989). Structured Clinical Interview for DSM-III-R-patient version (SCID-P, 9/1/89 version). New York: Biometrics Research Division/ New York State Psychiatric Institute.

Vikas, A., Avasthi, A., \& Sharan, P. (2011). Psychosocial impact of obsessive-compulsive disorder on patients and their caregivers: a comparative study with depressive disorder. Int $J$ Soc Psychiatry, 57, 45-56.

Westphal, A. C., Alonso, N. B., Silva, T. I., Azevedo, A. M., Caboclo, L. O. S. F., Garzon, E., Sakamoto, A. C., \& Yacubian, E. M. T. (2005). Comparação da qualidade de vida e sobrecarga dos cuidadores de pacientes com epilepsia por esclerose mesial temporal e epilepsia mioclônica juvenil. Journal of Epilepsy Clinical Neurophysiology, 11, 71-76.

Young, B., Dixon-Wood, M., Findlay, M., \& Heney, D. (2002). Parenting in a crisis: conceptualizing mothers of children with cancer. Social Science\& Medicine, 55, 1835-1847.

Zalihic, A., Markotic, V., Zalihic, D., \& Mabic, M. (2010). Gender and quality of life after cerebral stroke. Bosn J Basic Med Sci, 10, 94-99. 